\title{
Análise da pressão intra-ocular após ceratectomia fotorrefrativa com excimer laser
}

\author{
Intraocular pressure analysis after excimer laser photorefractive keratectomy
}

Christiane Rolim de Moura ${ }^{(1)}$

Aug'usto Paranhos Júnior (1)

Gustavo Teixeira Grottone ${ }^{(2)}$

Paula Boturão de Almeida ${ }^{(1)}$

Paulo Augusto de Arruda Mello ${ }^{(3)}$

João Antônio Prata Júnior ${ }^{(4)}$

\section{RESUMO}

Objetivo: Comparar os valores pré-operatórios da pressão intraocular (Po) com os valores pós-operatórios em pacientes submetidos à ceratectomia fotorrefrativa com excimer laser, bem como, avaliar os efeitos do uso de corticosteróide tópico na Po nesse grupo.

Pacientes e métodos: Foram analisados retrospectivamente 122 pacientes submetidos à ceratectomia fotorrefrativa com excimer laser para correção de miopia, no período de fevereiro de 1994 a dezembro de 1996. Esse grupo teve sua Po tomada com o tonômetro de aplanação de Goldmann no pré-operatório, $30^{\circ}$ e $60^{\circ}$ dias e sexto mês pós-operatório. Receberam cuidados pós-operatórios padronizados, incluindo o uso de corticosteróide tópico. Resultados: Foi observado um aumento estatisticamente significante em relação aos valores pré-operatórios nas médias da Po no $30^{\circ}$ dia pós-operatório, ao passo que no pós-operatório de seis meses obteve-se diminuição estatisticamente significante nos valores médios da Po em relação ao pré-operatório.

Conclusão: Nas fases iniciais do período pré-operatório, observou-se um aumento da Po possivelmente relacionado ao uso de corticosteróides. Após 6 meses, os valores da Po foram estatisticamente menores que os pré-operatórios.

Palavras-chave: Ceratectomia fotorrefrativa; Laser; Pressão intra-ocular.

\section{INTRODUÇÃO}

A ceratectomia fotorrefrativa com excimer laser é uma opção cirúrgica freqüente no tratamento da miopia. Sua influência na medida da pressão intra-ocular (Po) tem sido objetivo de algumas séries na literatura ${ }^{4}$. Recentes estudos tem mostrado uma redução na Po medida através de tonômetros de aplanação e de não contato após a cirurgia, que provavelmente, decorre de alterações da forma, estrutura e espessura da córnea secundárias ao procedimento cirúrgico ${ }^{4,7,8}$.

Outro fator que pode influenciar a Po é o uso de corticosteróides tópicos por longos períodos com o intuito de evitar a regressão do tratamento ou haze ${ }^{5}$. A incidência de pacientes "córtico-sensíveis" entre aqueles submetidos à ceratectomia fotorrefrativa com excimer laser que receberam corticosteróides tópicos no pós-operatório é referida entre 11 e 25\% . Entretanto, são raros os casos que necessitam de terapia antiglaucomatosa ou procedimentos fistulizantes com o intuito de levar a Po a níveis normais, pois a grande maioria retorna a essa situação após a interrupção do uso da medicação ${ }^{4,9}$. 
Sendo assim, esse estudo foi desenvolvido com o intuito de analisar a influência das alterações estruturais corneanas advindas da ceratectomia fotorrefrativa com excimer laser nas medidas da Po, bem como, observar o comportamento da Po durante a corticoterapia tópica em um grupo de pacientes míopes submetidos à cirurgia refrativa.

\section{PACIENTES E MÉTODOS}

Foram estudados, retrospectivamente, os prontuários de pacientes submetidos à ceratectomia fotorrefrativa com excimer laser para a correção de miopia e astigmatismo no setor de Cirurgia Refrativa do Departamento de Oftalmologia da UNIFESP - EPM, no período de fevereiro de 1994 a dezembro de 1996. Foram incluídos todos os casos submetidos à cirurgia, sendo considerado apenas um olho de cada paciente. Naqueles em que houve cirurgia bilateral, somente os dados do olho direito foram incluídos no estudo.

A ceratectomia foi realizada utilizando-se o Summit Apex Plus Excimer Laser ${ }^{\circledR}$. A escolha da área e profundidade tratadas baseou-se no erro refracional de cada paciente. Para a aplicação do tratamento, o epitélio era removido manualmente antes da ablação e os pacientes usaram lentes de contato terapêuticas até que a cicatrização epitelial estivesse completa (três a quatro dias). Nesse período utilizaram tobramicina associada a dexametazona $0,1 \%$ (Tobradex ${ }^{\circledR}$ - Alcon) ou ciprofloxacin associado ao mesmo corticóide (Biamotil-D ${ }^{\circledR}$ Frumtost) quatro vezes ao dia. Após essa fase, os pacientes receberam acetato de prednisolona $1 \%$ (Predfort ${ }^{\circledR}$ - Allergan) quatro vezes ao dia até o $20^{\circ}$ dia pós-operatório. A seguir foi indicada a administração de fluormetolona $0,1 \%$ (Flumex $0,1 \%^{\circledR}$ - Allergan) quatro vezes ao dia, do $21^{\circ}$ ao $60^{\circ}$ dia pósoperatório e duas vezes ao dia do $61^{\circ}$ ao $90^{\circ}$ dia pós-operatório. Após esse período os pacientes eram orientados a suspender a medicação.

Os dados demográficos, a refração, a área de ablação o número de pulsos e a Po foram coletados dos prontuários desses pacientes e registrados. A Po foi mensurada no pré e no pós-operatório (no $30^{\circ}, 60^{\circ}$ dia e sexto mês), por meio de um tonômetro de aplanação de Goldmann acoplado à lâmpada de fenda, sempre no período da manhã.

A fim de classificar os pacientes segundo sua resposta ao uso do corticosteróide tópico, estabeleceram-se critérios a partir daqueles previamente propostos por Armaly ${ }^{1,2}$. Os pacientes foram agrupados como não responsivos $(\geq 0 \mathrm{e}<2$ $\mathrm{mmHg}$ ), pouco ( $>2 \mathrm{e}<6 \mathrm{mmHg}$ ), medianamente $(\geq 6 \mathrm{e} \leq 15$ $\mathrm{mmHg}$ ) e altamente responsivos (>15 $\mathrm{mmHg}$ ). Na vigência de um aumento da Po do tipo altamente responsivo, que a equipe médica julgasse deletério ao paciente, o corticosteróide era suspenso e introduzida medicação antiglaucomatosa. Esses pacientes eram excluídos da amostra a partir do momento em que houvesse tal prescrição.

O teste de Friedman e o Método de Dunn foram utilizados para avaliar a significância da diferença entre as Po(s) medidas em cada intervalo e o teste de correlação de Spearman para se avaliar a correlação entre o valor esférico e o cilíndrico, a área de ablação, o número de pulsos e os valores da Po em cada intervalo ${ }^{6}$. O teste de Qui-quadrado foi utilizado para comparar a frequiência de indivíduos que não apresentaram aumento de Po secundário ao uso de corticóide tópico com a frequiência daqueles que foram considerados pouco e medianamente responsivos.

\section{RESULTADOS}

Foram estudados 122 olhos (103 olhos direitos e 19 olhos esquerdos) de 122 pacientes, sendo eles 67 do sexo feminino e 55 do sexo masculino. A idade média desse grupo foi de 31,5 anos $\pm 6,7$ (variando de 19 a 50 anos).

A refração esférica estática variou de $-15,25 \mathrm{D}$ a $+0,25 \mathrm{D}$ (média de $-4,81 \mathrm{D} \pm 3,03$ ) e a cilíndrica de $-0,75 \mathrm{D}$ a $-5,00 \mathrm{D}$ (média de $-1,10 \mathrm{D} \pm 0,90 \mathrm{D}$ ).

A área de ablação variou de 5 a $6,5 \mathrm{~mm}$ e o número médio de pulsos totais utilizado foi de 277,12 $\pm 119,77$ (mediana de 270,5, IC -95\%: 255,09, +95\%: 299,125).

Não se encontrou distribuição normal em nenhum dos intervalos pelo teste de Kolgomorov-Smirnov ( $\mathrm{p}<0,001)$. Por meio do teste de Friedman e do Método de Dunn observou-se um aumento estatisticamente significante nas médias da Po no primeiro mês do período pós-operatório em relação aos valores iniciais (Tabela 1). Após seis meses, observou-se diminuição estatisticamente significante nas médias da Po em relação aos valores pré-operatórios (Tabela 1).

$\mathrm{Na}$ Tabela 2, pode-se observar maior porcentagem de pacientes pouco e medianamente responsivos nos períodos de 30 e 60 dias. Tais diferenças foram estatisticamente significantes pelo teste do qui-quadrado ( $\mathrm{p}=0,002)$. Não se obteve pacientes altamente responsivos ao uso de corticosteróides

\begin{tabular}{|lccccccc|}
\hline \multicolumn{6}{|c|}{ Tabela 1. Estatística descritiva dos dados de Po em mmHg antes e nos vários intervalos após o procedimento, pelo Método de Dunn para } \\
se avaliar significância da diferença entre a Po antes e em cada intervalo.
\end{tabular}




\begin{tabular}{|c|c|c|c|c|c|}
\hline Período & $\begin{array}{l}\text { Sem resposta } \\
\leq 2 \mathrm{mmHg}\end{array}$ & $\begin{array}{c}\text { Pouco } \\
\text { responsivo } \\
>2 \mathrm{e}<6 \mathrm{mmHg}\end{array}$ & $\begin{array}{l}\text { Medianamente } \\
\text { responsivo } \\
\geq 6 \text { e } \leq 15 \mathrm{mmHg}\end{array}$ & $\begin{array}{l}\text { Altamente } \\
\text { responsivo } \\
>15 \mathrm{mmHg}\end{array}$ & $\mathbf{n}$ \\
\hline 1 mês & 48 (48,98\%) & 43 (43,88\%) & $07(07,14 \%)$ & - & 98 \\
\hline 2 meses & $58(57,42 \%)$ & $35(34,65 \%)$ & $08(07,92 \%)$ & - & 101 \\
\hline 6 meses & $59(77,63 \%)$ & $16(21,05 \%)$ & $01(01,31 \%)$ & - & 76 \\
\hline$P=0,002$ & & & & & \\
\hline
\end{tabular}

nesse grupo estudado. Alguns pacientes mantiveram Po elevada em relação aos valores pré-operatórios mesmo após a suspensão do corticosteróide. Dezesseis pacientes (21\%) mantiveram aumento dos níveis de Po entre dois e seis $\mathrm{mmHg}$ no sexto mês. Sete pacientes foram removidos do estudo pela necessidade de mudança em seus regimes terapêuticos (suspensão do corticosteróide e introdução de maleato de timolol 0,5\% para controle dos níveis pressóricos) a partir dessa alteração (Tab. 3). Até o momento da exclusão da amostra, esses pacientes não se comportaram com aumento de Po maior do que $15 \mathrm{mmHg}$ em relação ao período pré-operatório.

A correlação entre o valor da refração esférica, cilíndrica, área de ablação, número de pulsos e os valores de Po em cada intervalo não foi estatisticamente significante pelo teste de Spearman $(\mathrm{p}>0,05)$.

\section{DISCUSSÃO}

No presente estudo observou-se aumento nas médias de Po no intervalo de 30 dias pós-operatório. Esse período coincidiu com a fase em que os pacientes deixaram de usar acetato de prednisolona $1 \%$ para substituí-lo pela fluormetolona $0,1 \%$. No pós-operatório de 30 dias 35,91\% desenvolveram aumento entre dois e seis $\mathrm{mmHg}, 7,14 \%$ entre seis e $15 \mathrm{mmHg}$. Essas freqüências são maiores que as referidas em outros estudos da literatura e possivelmente possa ser justificada por diferenças nos critérios e definições de hipertensão ocular utilizados em cada estudo $4,7,8,9$.

Seiler e McDonnel descreveram pacientes que apresentaram níveis de Po acima de $40 \mathrm{mmHg}$ no pós-operatório de ceratectomia fotorrefrativa ${ }^{8}$. No presente estudo, sete pacientes apresentaram durante a evolução pós-operatória níveis pressóricos tais, que foram julgados deletérios pela equipe médica responsável, necessitando assim, de medicação antiglaucomatosa. Optou-se, dessa forma, por excluir esses pacientes do estudo, a partir do momento da mudança do regime terapêutico. Esse fato pode ter mascarado a presença de indivíduos altamente responsivos ao uso de corticosteróide tópico. Optou-se por essa conduta, pois o uso dessas medicações hipotensoras poderia influenciar nos valores finais e aumentar a porcentagem de pacientes com decréscimo da Po a longo prazo, o que poderia se sobrepor a um decréscimo na aferição da Po secundariamente às alterações estruturais corneanas do pós-operatório.

Neste estudo, detectou-se redução estatisticamente significante dos valores aferidos de Po aos seis meses de pósoperatório em relação àqueles pré-operatórios. Acredita-se que esse achado seja relacionado com alterações estruturais da córnea, entretanto a correlação entre a quantidade de pulsos, erro refracional ou área ablada com os níveis pressóricos nos diferentes intervalos, não foi evidente. Essa ausência de correlação estatisticamente significante também foi observada em outros estudos, exceto no estudo de Charttejee e col., no qual foi observada uma relação entre o decréscimo de Po a longo prazo nos olhos tratados e o erro refracional pré-operatório ${ }^{4,7}$. Deve-se salientar que o tamanho da amostra, o tipo de aparelho utilizado para realizar a ceratectomia, bem como para aferir a Po e a análise estatística diferiram significativamente entre o estudo citado e o presente estudo.

Por outro lado, deve-se ressaltar que a diferença entre os valores medianos pré e pós-operatórios foi de dois $\mathrm{mmHg}$, o que clinicamente não parece ser significante.

Com base nos dados deste estudo, pode-se observar que no $30^{\circ}$ dia pós-operatório ocorreu aumento da Po devido à sensibilidade aos corticosteróides e a longo prazo os valores de Po são inferiores aos pré-operatórios. Esse último resultado possivelmente está relacionado a alterações estruturais da córnea,

\begin{tabular}{|c|c|c|c|c|c|c|}
\hline & Média & DP & Mediana & IC -95\% & IC +95\% & $\mathbf{N}$ \\
\hline Pré-operatório & 18,70 & 4,39 & 17,00 & 4,06 & 6,15 & 7 \\
\hline 1 semana & 23,00 & 4,58 & 22,00 & 11,36 & 25,88 & 3 \\
\hline 1 mês & 19,50 & 5,86 & 18,50 & 6,15 & 9,64 & 6 \\
\hline 2 meses & 19,50 & 3,08 & 20,50 & 3,23 & 5,07 & 6 \\
\hline 6 meses & 15,30 & 4,31 & 15,00 & 3,99 & 6,04 & 7 \\
\hline
\end{tabular}


as quais comprometem a acurácia da tonometria de aplanação. No entanto, essas diferenças de valores não parecem ser significantes do ponto de vista clínico.

Agradecimentos: Os autores agradecem ao Setor de Cirurgia Refrativa da UNIFESP - EPM, pelo acesso aos seus arquivos.

\section{SUMMARY}

Purpose: To compare the preoperative and postoperative values of IOP in a group of patients after photorefractive keratectomy (PRK) and to evaluate the sensitivy to steroid drops.

Methods: One hundred and twenty-two patients who underwent PRK from February 1994 to December 1996 were analized retrospectively. IOP measurements were taken with a Goldmann applanation tonometer preoperatively and postoperatively on the $30^{\text {th }}, 60^{\text {th }}$ and $180^{\text {th }}$ day. All of them received the same postoperative routine, including steroid drops.

Results: Comparing with the preoperative values, there was a statistically significant increase in IOP on the 30th postoperative day, while there was a statistically significant decrease in IOP at the 6th month.

Conclusions: On the $30^{\text {th }}$ postoperative day, IOP showed a statistically significant increase probably due to the use of steroid drops. Six months IOP showed a statistically significant decrease in relation to preoperative values.

Keywords: Photorefractive keratectomy; Laser; Intraocular pressure.

\section{REFERÊNCIAS BIBLIOGRÁFICAS}

1. Armaly MF. Statistical attributes of the steroids hipertensive response in the clinically normal eye I. The demonstration of three level response. Invest Ophthalmol 1965;4:187.

2. Armaly MF. Effect of corticoids on intraocular pressure and fluid dinamics I. The effects of dexamethasone in the normal eye. Arch Ophthalmol 1963;70:88.

4. Chatterjee A, Shah S, Bessant D, Doyle S. Reduction in intraocular pressure after Excimer Laser Photorefractive Keratectomy. Ophthalmology 1997; 104:355.

5. Gastry DS, Kerr Muirr M, Marshal J. The effects of topical corticosteroids on refraction and corneal haze following Excimer Laser treatment for myopia: a prospective, randomized, double-masked study. Eye 1993;7:584

6. Glantz AS. Alternative to analysis of variance and the t test based on ranks. In - Primer of bio-statistics. $4^{\circ}$ ed. New York, McGraw-Hill, 1997;323-72.

7. Mardelli PG, Piebenga LW, Whitacre MM, Siegmund KD. The effect of excimer laser photorefractive keratectomy on intraocular pressure measurements using the Goldmann applanation tonometer. Ophthalmology 1997; 104:945.

8. Seiler T, McDonnel P. Excimer Laser Photorefractive Keratectomy. Surv Ophthalmol 1995;40:89.

9. Skuta GL, Morgan RK. Corticosteroid-induced Glaucoma. In: Ritch R, Shields B, Krupin T.: The Glaucomas - 2nd. edition, Mosby, Saint Louis, MO, 1996;1177. 